\title{
Digital transformation routes, data mining methods as a preliminary stage of predictive analytics in corporate finance
}

\author{
Alla A. Nikonova ${ }^{1}$, Elena V. Krasil'nikova ${ }^{2}$ \\ The Central Economics and Mathematics Institute of the Russian Academy of Sciences \\ Moscow, Russia \\ ${ }^{1}$ Prettyal@cemi.rssi.ru, ${ }^{2}$ Krasilnikova_lena@list.ru
}

\begin{abstract}
We focus on several areas of digital transformation. In particular, the possibility of applying predictive analytics in corporate finance is the key area for this paper. We have built probability models, which forecast a company moving along a life cycle curve. Also, these models are able to predict instability during company's life cycle. The models are based on logistic regression, which is one of the key algorithms of data mining. Relying on our own survey conducted at a microelectronics plant, the lack of digital elements in the estimation of investment projects has been found. The hypothesis about the transformation of corporate relations has been formulated. In the future, this hypothesis may help to modify the theory of digital entrepreneurship.
\end{abstract}

Keywords: digital, predictive analytics, data mining in corporate finance, enterprises development.

\section{INTRODUCTION}

Sustainable economic development strategies should consider the opportunities and limitations of the updated technological base. The Third Industrial Revolution is associated with computer numerical control and microprocessors. Innovations that contributed to a technological breakthrough until 2000s are no longer relevant for the challenges of the Fourth Industrial Revolution. The purpose of this Industrial Revolution is to enhance competitiveness through the implementation of cyberphysical systems in production. At present, some elements of digitalization are being actively used. There are significant opportunities for improvement of financial performance and increase returns on digital investments in all sectors of industry. Using technological innovation and broadening the opportunities for applying digital advances facilitates economic sustainability. Except for quantitative factors and determinants, economic revolution is impossible without structural transformations. According to J. Schumpeter, such changes are related to "creative destruction", when technological innovation destroys the traditional workplace [1]. Enterprises should consider the digital technology not only in terms of costs and focus on a source of economic and competitive advantage. By its nature, the technology industry accumulates innovation by providing technological infrastructure that boosts the growth of the economy as a whole.

Referring to A. Trachuk, The Fourth Industrial Revolution is pervasive, covers technological changes, institutional transformation, lays the foundation for a number of policy initiatives and furthermore facilitates the development of digital culture in society [2].

\section{TECHNOLOGICAL DIGITAL CHANGES IN THE WORLD AND INRUSSIA}

A. World trends

According to the World Digital Competitiveness Ranking in 2018 and 2019 the leaders are: United States, Singapore, and Sweden [3]. For example, the largest investment was attracted in FinTech industry in UK, even so it is ranked on 10th position in 2018 and 15th in2019. For Russia, an increase in some elements has been found to the criterion of the technological base: patents, the development of information and communication technologies (ICT). However, according to the overall rating of Digital Competitiveness, Russia takes 38th place in 2019, 40th place in 2018, 42nd place in 2014. Referring to The Boston Consulting Group Russian economy has the capabilities to grow the digital economy from $2.1 \%$ of Gross Domestic Product (GDP) to $5.6 \%$ by 
2020. The program of the National Technology Initiative is actively developing, the main goal is - buildup conditions for global technological leadership. Mostly large Russian companies are moving toward digital transformation. The problems were identified in the lack of comprehensive (complexity) programs and separate digital solutions on practice.

\section{B. Transition from Traditional approaches to maintaining Predictive Analytics}

Enterprises development forecasts are based on models that well describe the current performance, identify the reasons and relations of historical data changes. In tested models, the concept of enterprise stability and instability is often used, which is directly related to the financial and economic results. Financial and economic sustainability of the enterprise is the position, in which the distribution and use of resources ensures development on the basis of profit and capital growth, maintaining solvency of an acceptable level of risk [4]. This requires special flexibility in the structure of financial resources, creditworthiness and investment attractiveness. For example, it is rather problematic to track the effects of changes in stock prices on performance indicators, due to velocity. Real-time forecasts and predictions estimated by business intelligence tools, improve the relevance results, model significance and allow timely response to deviation shocks. Due to the high level of information technology (IT) tools and instruments, today analysts in companies deal with the processing of huge volume of variety and velocity data, which is a rather labor-intensive process. Conceptual frameworks of analytics are changing, business models are being modified. Further evolution is taking place: from computer technologies, applications and spreadsheets, tools to reporting, using IT in creating value - to big data, digital entrepreneurship. Based on Eurostat data (there is no USA in the sample) it was found that the analysis of big data is mainly provided by enterprises employees and not by third-party analysts. This characterizes the positive trend of advanced digital knowledge and skills (Fig. 1).

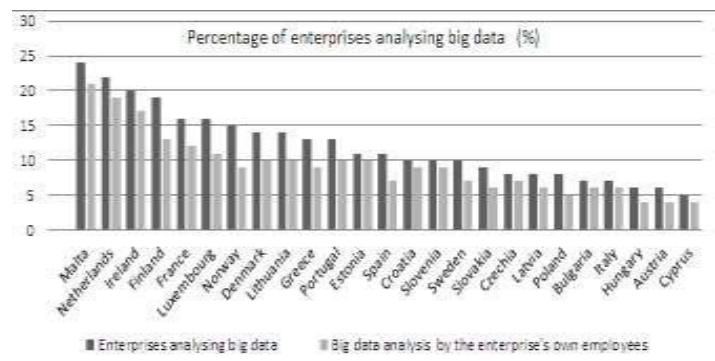

Fig. 1. Big data analysis in enterprises [5]

Cloud computing, predictive analytics and cognitive computing create the greatest value for their companies. Deloitte research defined that a half of Chief Financial Officers have already invested in advanced financial and accounting analytics, and 52\% will continue investment to improve forecasting [6]. In many Russian companies even the previous generations technologies like systems of computer production management, electronic document management, automation of managerial and accounting, planning and supply chain management are poorly implemented [2]. On the one hand, this indicates a lack of readiness for changes; on the other hand, there are opportunities to increase efficiency. The traditional analysis in corporate finance focuses on construction and estimation of sustainability. The possibility of long-term stability of the company based on the analysis of financial statements, determination trends in financial ratios, liquidity, asset turnover, debt management, profitability, market value. Investment and financial analysts in real Russian companies usually use own calculations in Microsoft Excel, Visual Basic for Applications, software packages like Stata, Eviews to create financial models. Determining the long -term development 
and sustainable growth of the company is possible through the use of complex approach. It includes the systematization and characteristics the stages of life cycle and conclusions from models that find impacts on the financial and economic performance indicators. The relation of financial and economic factors, company performance can be determined based on regression analysis models. But in most cases such models ignore the characteristics and adjustments to the stage of life cycle, which drastically affects the results. The companies are not static, preferences and sources of funding vary at different stages. Features for mature companies differ from the characteristics for growing enterprises. The life cycle concepts mostly provide a description of changes just in organizational characteristics, missing an impact on financial and economic factors. We built models that describe the company's transition along its life cycle curve. Logistic regression models were constructed based on panel historical data of public Russian companies. They find out the impact on factors on the company's stage of life cycle and also simulate the probability. The components of predictive analytics include: data collection, application of analysis methods and further forecast. Logistic regression is one of instruments or algorithms of data mining. The resulting model can be trained to make predictions. According to the conclusions of the analytical researches, the difficulty of raising funds by Russian enterprises is a key issue and causes underinvestment of high-tech industries in unstable economy, especially in credit and financial environment decline. The results of testing of economic and mathematical models have shown that young companies mainly use internal sources, raise bank loans at a mature stage. Structuring potential sources of capital for every level of the organization can help support its sustainable growth. Macroeconomic situation has a dominant impact in the conditions of instability, in contrast to the prevailing influence of corporate relations with the growth of the economy. In a situation of economic recession, level of debt capital is reduced in relation to the company's assets.

\section{Equations}

We assume different strategies depending on the stage of development.

$$
\begin{aligned}
& f(z)=P\left(Y_{i t}=1 \mid X_{i}\right) ; \\
& Z_{i t}^{*}=\beta^{*} X_{t}+\varepsilon_{i} ; \\
& f(z)=\frac{1}{1+e^{-Z}},
\end{aligned}
$$

$y$ - dependent binary variable; $f(Z)$ - logistic function; $Z_{i t}-$ linear combination of independent variables; $x_{i t}$-independent variable; $\varepsilon_{\mathrm{it}}$ - error; $t=1 \ldots T_{i}, i=1 \ldots n$. The following variables were explored: operating profit margin, revenue, market capitalization, number of employees, current ratio, financial leverage (assets divided by equity, that equal debt divided to equity plus one), industry (dummy variable).

First hypothesis: at each life cycle there is a different impact of financial and economic factors, in particular, financing indicators. Identifying transition drivers from one life cycle stage to another promotes the sustainable development of the company, modifies corporate governance based on founded characteristics, decreases hypothetical obstacles to company's development. According to the results of our own simulation, the probability determinants defined scenarios of potential development of the company. The obtained signs at the coefficients of explanatory variables based on logistic regressions characterize the probability: a positive coefficient indicates an increase in the probability of transition to a new stage of the life cycle and negative - a decrease. We obtained positive effect of financial leverage and the negative impact of operating profit margin on the probability of a transition from the stage of slow growth to the stage of rapid growth, which is illustrated in the Figure 2. The probability to turn to rapid growth stage is increasing by $5.6 \%$ due to rise in financial leverage. 


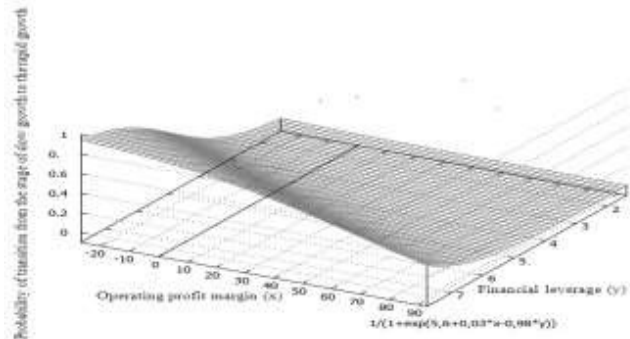

Fig. 2. Three-dimensional graph modeling the probability of transition from slow growth to the stage of rapid growth

Active debt raising is an orderly process for growing enterprises. The negative effect of leverage increase on the probability of transition to the maturity stage has been revealed. That is, if a company is actively increasing its debt on growth stage, this reduces chances of moving into the maturity stage. We estimated the fall probability by $22 \%$. So, one simple recommendation: significant debt growth can reduce the probability of a company becoming mature and trigger recession. The market capitalization rise also reduces the company's chances to move from the stage of rapid growth to the stage of maturity, implying that the highest stock prices have the most growing companies. Also do not underestimate the impact of the macroeconomic situation: with an increase in the GDP, the company's probability of moving from a stage of slow growth to a stage of rapid growth will increase by $1.2 \%$; with an increase in financial leverage, the company's probability of moving to a stage of rapid growth will increase by $14.3 \%$. Another objective of this study is devoted to explore factors related to the probability of instability. To companies at the recession stage or with operating profit margin decline (due to a decrease in sales and an increase in costs) probability of unsustainable growth is raising. On the basis of probability research methods it is proved that overinvestment reduces the company's chances of remaining at the maturity stage. Thus, the development and growth of the company is presented as complex hierarchical processes that should be explored in the context of several subsystems of different levels of the economic hierarchy. Management should apply the results of probability simulation to create forecasts and scenarios about the prospects of company's development, potential threats and incentives. The predictive analytics precisely helps to timing reaction and changes in volatile and velocity factors influenced the sustainability of enterprises. Based on our brief surveys of analysts at the microelectronics plant in Moscow region, were obtained key points of evaluating investment projects and the estimation financial and economic position. In corporate finance department, digital economy tools are poorly applied.

The role of advanced analytics in corporate finance is slightly underestimated. So, according to KPMG survey, about $50 \%$ large companies only test advanced analytics methods and just $7 \%$ has used [7]. For Russian enterprises with problems in the field of technology but have growth drivers in digital skills, new strategies should be based not only on the quantitative buildup and implementation of technologies, but on the overall system transformation.

\section{DIGITAL AND AGENCY RELATIONS}

According to the Russian Government Program, the digital economy consists of three levels: markets and industries, the interaction of economic agents; platforms and technologies; institutional environment, regulation, human resources, information infrastructure and security. Digital revolution is not just another technological advancement, but the transformation of all the basic functions, mechanisms, frameworks. Changes in the interactions of stakeholders promote new corporate culture. All this transform the traditional problems of the principal-agent, managers 
and directors, managers and owners. With the appliance of digital technologies, the asymmetry of transactions becomes narrow, and confidence increases. This reduces the traditional opportunism of economic agents. The main problems can face with human capital: brain drain, lack of specialists and rapid transformation of the traditional background. Key figures can be financial leaders who are agile and flexible. They have to find a balance and compromise between standardization and experimentation, create roles and decision-making rights for digital events. They should develop technological thinking and skills of data analytics. Thus, it is often mistakenly assumed that the digitalization of the economy is based only on new technologies. The main obstacles are associated with the underdevelopment of digital culture, the inability of companies to transform rapidly and the treat of survival for small and medium-sized enterprises in the face of radical changes.

The task of transition to a digital economy set by the Government of Russia is controversial. We have built probability models for determining the prospects for the development of the company based on logistic regression model. The results of this economic-mathematical simulation present the significant role of factors in the context of the study of sustainable development. Logistic regression is an essential component of predictive analytics. Besides that, it is also one of the key algorithms of data mining. Changing in stock prices is a fairly velocity process. It takes time to track and evaluate the impact of changes. Predictive analytics tools enable to achieve instant results. Using these methods contributes to the corporate tasks and qualitative strategies. On the other hand, significant institutional and social characteristics and problems can be ignored in order to increase quantitative criteria for the introduction of digital technologies. Not all enterprises are ready for such changes, which can lead to differentiation and non-survival. This transition signifies a drastic transformation, both at the micro and global national strategies level. The major challenge is to rethink the traditional processes: internal and stakeholder relations, business operations and models. Future research will be related to the modification of the theoretical concepts of agency relationships, digital entrepreneurship, and practical application of advanced analytics methods to enhance forming corporate strategies.

\section{REFERENCES}

[1]. Schumpeter J. Capitalism, socialism and democracy. 2nd ed. Floyd,: Impact Books, 2014.

[2]. Trachuk A., Linder N., Tarasov I. et al. Transformation of industry in the conditions of the Fourth Industrial Revolution. St. Petersburg: Real Economy, 2018.

[3]. IMD World Digital Competitiveness Ranking 2019. Available at: https://www.imd. org/wcc/world-competitiveness-center-rankings/worlddigital-competitiveness-rankings-2019.

[4]. Rodionova V., Fedotova M. The financial stability of the enterprise in terms of inflation. Moscow: Perspective, 1995.

[5]. Eurostat. Available at: http://ec.europa.eu/eurostat/data/database.

[6]. Crunch time. Finance in a digital world. Deloitte, 2016. Available at: https://www2.deloitte. $\mathrm{com} / \mathrm{content} / \mathrm{dam} /$ Deloitte/global/Document s/Finance/gx-finance-crunch-time-financein-a-digital-world.pdf.

[7]. Digital technologies in Russian companies. KPMG, 2019. Available at: https://assets. $\mathrm{kpmg} /$ content/dam/kpmg/ru/pdf/2019/01/ru-ru-digitaltechnologies-in-russian-companies. pdf. 